\title{
Choque séptico con disfunción multiorgánica en paciente con pericarditis supurada $y$ bacteriemia por Staphylococcus aureus meticilino sensible. Reporte de caso
}

Septic shock and multi organ dysfunction in a patient with purulent pericarditis and bacteremia by methicillin-sensitive Staphylococcus aureus. A case-report

Jaime Zegarra',a, Vanessa Saavedra ${ }^{1, a}$, Luis Granados ${ }^{1, b}$, Erika Rojas ${ }^{1, a}$, Sandra Yucra ${ }^{1, c}$, Antonio Hernández ${ }^{2, b}$

\section{RESUMEN}

Choque séptico con disfunción multiorgánica tiene una tasa de mortalidad mayor de $50 \%$. Se presenta el caso de un varón que cursó con choque séptico con disfunción multiorgánica, secundario a bacteriemia por Staphylococcus aureus meticilino sensible, con foco de partida dérmico - partes blandas, quien presentó lesiones alveolo nodulares, pericarditis supurativa, lesiones embolicas a nivel dérmico, ocular y renal; recibió soporte en la unidad de cuidados intensivos, tratamiento antibiótico con oxacilina más vancomicina, pericardiectomia subtotal y drenaje pleural. Salió de alta estable para continuar tratamiento. Presentamos este caso para destacar la gravedad de la bacteriemia por Staphylococcus aureus meticilino sensible, con compromiso multiorgánico y así poder tomar medidas terapéuticas agresivas para disminuir la morbilidad y mortalidad por el mismo.

PALABRAS CLAVE: Choque séptico, insuficiencia multiorgánica, pericarditis, bacteriemia, supuración, infecciones estafilocócicas. (Fuente: DeCS BIREME).

\section{SUMMARY}

Septic shock with multi organ dysfunction is associated with a mortality above $50 \%$. We present here the case of a young male patient who presented with septic shock and multi organ dysfunctions secondary to methicillinsusceptible Staphylococcus aureus from a skin and soft tissue infection presenting with alveolar-nodular pulmonary lesions, purulent pericarditis and septic embolic lesions on the skin, eyes and kidneys. The patient was admitted to the ICU receiving antibiotic coverage with oxacillin and vancomycin, subtotal pericardiectomy and pleural drainage, and was discharged clinically stable. We present this case to draw attention to the severity of S. aureus bacteremia to initiate prompt aggressive therapeutic measures to ameliorate associated morbidity and mortality.

KEYWORDS: Septic shock, multi-organ failure, pericarditis, suppuration, bacteremia, staphylococcus infections. (Source: MeSH NLM).

\footnotetext{
Unidad de cuidados intensivos, Hospital Cayetano Heredia. Lima, Perú.

Departamento de Medicina, Hospital Cayetano Heredia. Lima, Perú.

Médico asistente;

Médico Jefe;

Médico residente
} 


\section{INTRODUCCIÓN}

Choque séptico con disfunción multiorgánica tiene una tasa de mortalidad mayor de $50 \%$. Los agentes etiológicos son bacterias Gram positivas, Gram negativas y hongos; el foco más frecuente es pulmonar, seguido de gastrointestinal, genitourinario y partes blandas (1).

Se presenta el caso de un varón que cursó con choque séptico con disfunción multiorgánica, secundario a una bacteriemia por Staphylococcus aureus meticilino sensible, con foco de partida dérmico/partes blandas, quien cursó con lesiones alveolares nodulares, pericarditis supurativa, lesiones embólicas a nivel dérmico, ocular y renal. El paciente recibió manejo y soporte en la unidad de cuidados intensivos (UCI), tratamiento antibiótico con oxacilina más vancomicina, pericardiectomia subtotal más drenaje pleural. Salió de alta estable para continuar tratamiento.

El objetivo de la presentación de este caso fue destacar la gravedad de la bacteriemia por Staphylococcus aureus meticilino sensible, con compromiso multiorgánico, y así tomar medidas terapéuticas agresivas para disminuir la morbilidad y mortalidad por el mismo.

\section{PRESENTACIÓN DEL CASO}

Varón de 26 años; diez días antes del ingreso cursó con dolor lumbar, seguido de lesiones pápuloeritematosas en la cara externa de la pierna izquierda, por lo cual recibió tratamiento con diclofenaco, vitamina $\mathrm{B}_{12}$, tramadol, dexametasona, clorfeniramina y paracetamol, sin presentar mejoría. Ingresó por emergencia al Hospital Cayetano Heredia con fiebre, ictericia, disnea; al examen físico se encontró PA:
80/60 mm Hg; FC: 134 x min, FR: 22 x min, T: $37^{\circ} \mathrm{C}$, Sat $0_{2}$ : $97 \%$; en mal estado general, edema en miembros inferiores, lesiones pápulo-eritematosas en la cara externa de pierna izquierda, una con secreción purulenta escasa, subcrepitantes en las bases pulmonares, ingurgitación yugular $(+)$, soplo sistólico II/IV en mesocardio, frote pericárdico $(+)$, dolor abdominal en hipocondrio izquierdo, Glasgow 15.

La radiografía de tórax mostró patrón intersticial y cardiomegalia; el electrocardiograma, taquicardia sinusal, elevación difusa del segmento ST compatible con pericarditis (figura 1), hemograma con leucocitos $34,380 \times \mathrm{mm}^{3}$, abastonados $10 \%$, urea $77 \mathrm{mg} /$ $\mathrm{dl}$, creatinina $1,4 \mathrm{mg} / \mathrm{dl}$, bilirrubina total $1,9 \mathrm{mg} /$ dl, plaquetas $90000 \times \mathrm{mm}^{3}$, INR: 1,23; lactato 1,7 $\mathrm{mmol} / \mathrm{l} ; \mathrm{PaO}_{2} / \mathrm{FiO}_{2}: 314$.

Recibió manejo inicial de choque con cloruro de sodio al $0,9 \%$ e infusión de noradrenalina. A las 24 horas presentó deterioro hemodinámico y respiratorio requiriendo intubación endotraqueal y ventilación mecánica invasiva. Ingreso a la UCI con apoyo ventilatorio e infusión de noradrenalina $0,11 \mathrm{ug} / \mathrm{kg} /$ min; el score APACHE II fue 25, SOFA 12; presión arterial media (PAM) $64 \mathrm{~mm} \mathrm{Hg}$, FC $106 \times$ min, $\mathrm{Sat0}_{2}:$ 96\%, PVC $16 \mathrm{~mm} \mathrm{Hg}$; Satv0 : 81,8\%, llenado capilar $>3$ segundos (tabla 1), con oliguria, cianosis, hemorragias en astillas en lecho ungueal y manchas de Roth en el fondo de ojo (figura 2 y 3 ); índice cardiaco (IC) por Fick 6,02; resistencia vascular sistémica (RVS) 384 dinas $/ \mathrm{seg} / \mathrm{m}^{2}$. Se realizó ventilación mecánica con volumen tidal $6 \mathrm{ml} / \mathrm{kg}$ de peso ideal, con presión plateau $27 \mathrm{y}$ driving pressure 15.

La ecocardiografía mostró fracción de eyección de $30 \%$, hipoquinesia global, efusión pericárdica leve, no lesiones valvulares ni vegetaciones; el monitoreo con

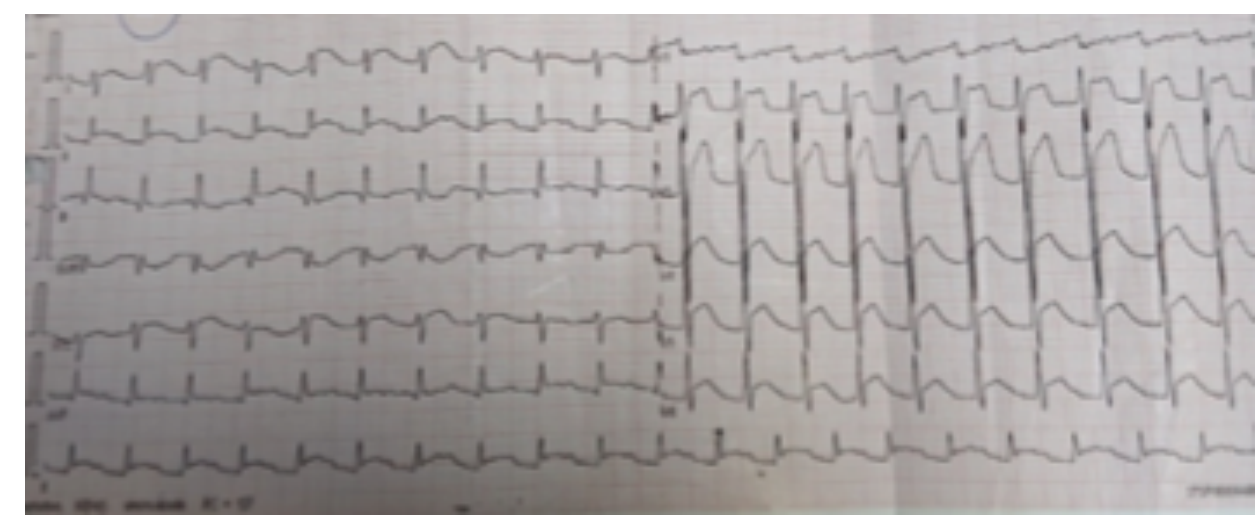

Figura 1. Electrocardiograma, muestra taquicardia sinusal y elevación difusa del segmento ST. 


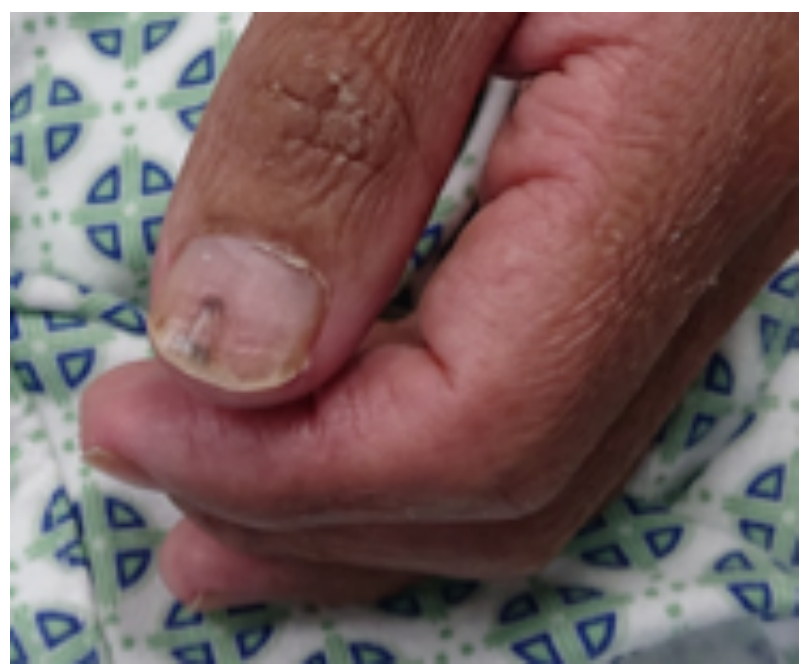

Figura 2. Hemorragias en astillas en el lecho ungueal.

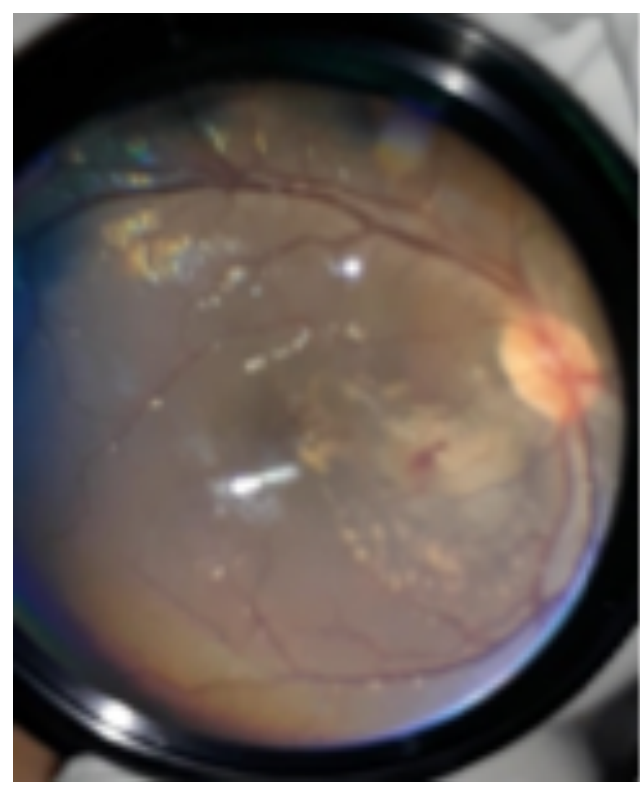

Figura 3. Fondo de ojo: manchas de Roth.
Doppler esofágico mostró IC: 3.1; RVS 760 dinas/seg/ $\mathrm{m}^{2}$; tiempo de flujo corregido (TFc) 272; transporte de oxigeno (D02) 760, consumo de oxigeno (V02) 139 (tabla 1).

El hemograma mostró reacción leucemoide, plaquetopenia, aumento de ácido láctico y azoemia (tabla 2); se aisló Staphylococcus aureus meticilino sensible (MSSA) en dos muestras de hemocultivo, en cultivo de secreción bronquial y del líquido pericárdico. La tomografía espiral multicorte de tórax (TEM) reveló lesiones alveolo-nodulares bilateral (figura 4), la TEM de abdomen y pelvis, hepatoesplenomegalia y ascitis; en la fibrobroncoscopia no se encontraron hallazgos de importancia. En el manejo de choque se requirieron dosis altas de noradrenalina, adrenalina y vasopresina (tabla 3 ).

La ecocardiografía control a las 72 horas evidenció efusión pericárdica severa de aproximadamente 600 $\mathrm{ml}$, con ecos refringentes, hipoquinesia global (figura 5 y figura 6); por lo cual se realizó pericardiectomia subtotal con drenaje de $500 \mathrm{ml}$ de líquido pericárdico purulento, más drenaje de empiema. En informe anátomo-patológico indicó pericarditis con infiltrado inflamatorio agudo y crónico, parénquima pulmonar con extensa lesión necrótica y supurativa compatible con neumonía necrotizante. El tratamiento antibiótico inicial fue oxacilina + vancomicina, y luego de los resultados de los cultivos, se des escaló a oxacilina.

La evolución fue con persistencia de la fiebre y ante la sospecha de endocarditis infecciosa se realizó ecocardiografía transesofágica, no encontrándose vegetaciones ni lesiones valvulares. En una nueva muestra de hemocultivo se aisló Klebsiella pneumoniae metalobetalactamasa positivo $(\mathrm{kpc})$, indicándose

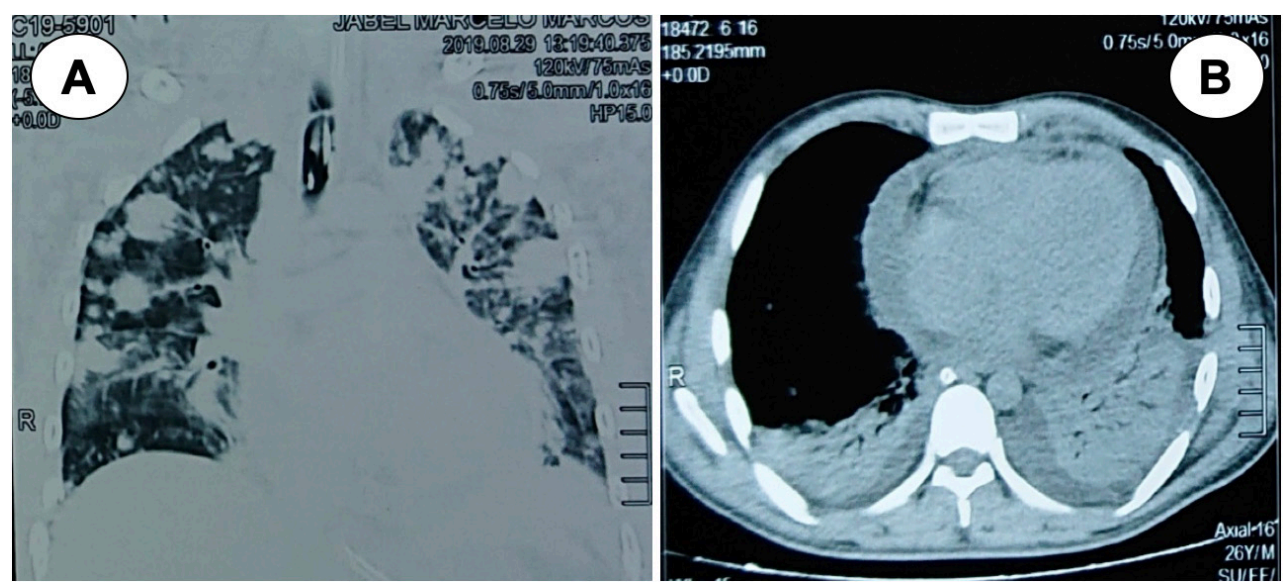

Figura 4. TEM tórax. A: Lesiones alvéolo-nodulares pulmonares bilateral y cardiomegalia. B: Muestra compromiso pleuro-parenquimal bilateral y efusión pericárdica. 
Tabla 1. Parámetros clínicos, hemodinámicos y ventilatorios.

\begin{tabular}{|c|c|c|c|c|c|}
\hline Parámetro & día 1 & día 3 & día 5 & día 7 & final \\
\hline PAM (mm Hg) & 64 & 70 & 80 & 90 & 85 \\
\hline $\mathrm{FC} x \min$ & 106 & 140 & 123 & 89 & 88 \\
\hline PVC (mm Hg) & 16 & 16 & 14 & 22 & \\
\hline $\mathrm{Sat0}_{2}(\%)$ & 96 & 98 & 98 & 98 & 97 \\
\hline $\operatorname{Satv}_{2}(\%)$ & 81,8 & 83 & 81 & 81 & \\
\hline Lactato $(\mathrm{mmol} / \mathrm{l})$ & 1,5 & 3,2 & 2,6 & 4,5 & 1,2 \\
\hline $\mathrm{PaO}_{2} / \mathrm{FiO}_{2}$ & 288 & 292 & 293 & 247 & 330 \\
\hline \multicolumn{6}{|l|}{ Doppler esofágico } \\
\hline Índice cardiaco & 6,03 & 3,1 & 2,6 & 2,7 & \\
\hline RVS (dinas/seg/m²) & 384 & 760 & 1025 & 995 & \\
\hline $\mathrm{TFc}$ & & 272 & 286 & 297 & \\
\hline Volumen sistólico & 104 & 40 & 53 & 54 & \\
\hline D02 & 1498 & 779 & 640 & 681 & \\
\hline V02 & 217 & 130 & 110 & 117 & \\
\hline \multicolumn{6}{|l|}{ Ecocardiografía } \\
\hline Fracción de eyección (\%) & 30 & 38 & & & \\
\hline Efusión pericárdica (ml) & & 600 & & & \\
\hline \multicolumn{6}{|l|}{ Mecánica ventilatoria } \\
\hline Volumen tidal espirado & 620 & 431 & 517 & 576 & \\
\hline PEEP & 10 & 9 & 10 & 8 & \\
\hline Presión plateau & 22 & 22 & 23 & 22 & \\
\hline Driving pressure & 12 & 13 & 13 & 14 & \\
\hline Compliance estática & 51 & 35 & 40 & 41 & \\
\hline
\end{tabular}

Tabla 2. Exámenes de laboratorio.

\begin{tabular}{lrrrrr}
\hline Parámetro & \multicolumn{1}{c}{ día 1 } & \multicolumn{1}{c}{ día 3 } & día 5 & \multicolumn{1}{c}{ día 7 } & \multicolumn{1}{c}{ final } \\
\hline Leucocitos $\mathrm{x} \mathrm{mm}^{3}$ & 46350 & 35610 & & 31790 & 11000 \\
Plaquetas $\mathrm{x} \mathrm{mm}^{3}$ & 99000 & 140000 & & 223000 & 250000 \\
$\mathrm{INR}$ & 1,26 & 1,39 & & 1,36 & 1,1 \\
Creatinina $(\mathrm{mg} / \mathrm{dl})$ & 0,67 & 2,7 & 1,5 & 1,84 & 0,9 \\
Bilirrubina $(\mathrm{mg} / \mathrm{dl})$ & 7,2 & & 2,2 & & 1,5 \\
Proteinuria orina 24 h & & & 1,0 & & \\
\hline
\end{tabular}

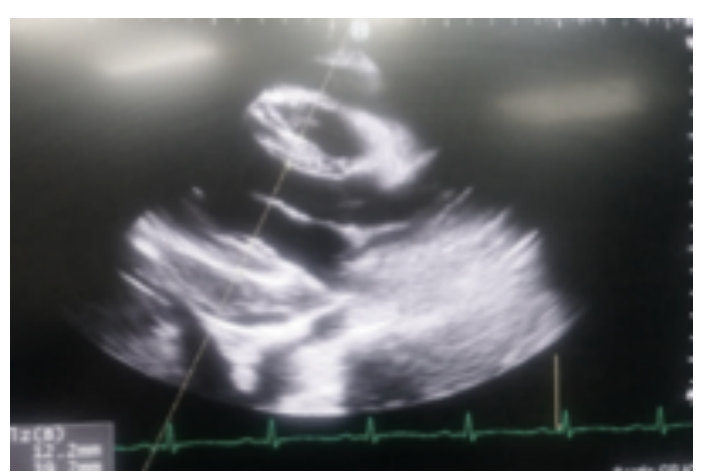

Figura 5. Ecocardiografía: efusión pericárdica colistina; asimismo, por la persistencia de la colección pleural se realizó re - intervención quirúrgica con liberación del parénquima pulmonar, decorticación parcial y drenaje de empiema $(500-600 \mathrm{ml})$, con lo cual la evolución fue favorable, siendo dado de alta de la UCI.

\section{DISCUSIÓN}

Se presenta el caso de un varón que cursó con choque séptico más disfunción multiorgánica (DMO), secundario a bacteriemia y pericarditis supurada por 
Tabla 3. Tratamiento.

\begin{tabular}{|c|c|c|c|c|c|}
\hline Parámetro & día 1 & día 3 & día 5 & día 7 & final \\
\hline Noradrenalina (ug/kg/min) & 0,15 & 0,50 & 0,34 & 0,13 & \\
\hline Adrenalina (ug/kg/min) & & 0,04 & & & \\
\hline Vasopresina (UI/min) & & 0,04 & 0,04 & & \\
\hline \multicolumn{6}{|l|}{ Antibióticos } \\
\hline Oxacilina & + & + & + & + & + \\
\hline Vancomicina & + & + & + & + & \\
\hline Colistina & & & & + & + \\
\hline \multicolumn{6}{|l|}{ Quirúrgico } \\
\hline Pericardiectomia subtotal & & & + & & \\
\hline Drenaje pleural/decorticación & & & & + & \\
\hline
\end{tabular}

Staphylococcus aureus meticilino sensible (MSSA), que tuvo como punto de partida infección de partes blandas. Por la historia y la evaluación clínica, el foco de partida de la bacteriemia fue piel/partes blandas.

Lodise (2), reporta que el punto de partida más frecuente para bacteriemia por estafilococo meticilino resistente (MRSA) fue infección vascular y piel / tejidos blandos. Jiun-Ling Wang (3), reporta casos de bacteriemia por MRSA secundaria a infección de partes blandas y neumonía necrotizante (3).

La bacteriemia e infecciones por gérmenes Gram positivos son las causas más frecuentes de choque séptico con DMO, siendo la mortalidad de más del $50 \%$ (4-7). Los hallazgos clínicos tipo hemorragias en astillas en el lecho ungueal, manchas de Roth en el fondo de ojo, proteinuria significativa en la orina de 24 horas (8), compromiso pulmonar con lesiones alveolo nodulares bilateral, pericarditis supurada, fueron manifestaciones de la bacteriemia por MSSA (9). Las lesiones alveolo nodulares y el hallazgo anátomo patológico de neumonía necrotizante que tuvo nuestro paciente han sido descritas en estudios previos. Wesley H. Self (10), reporta que estafilococo aureus es la segunda causa bacteriana de neumonía comunitaria, después de estreptococo pneumoniae, los pacientes al ingreso cursaron con infección concurrente por influenza, hemoptisis e infiltrado alveolo nodular multilobar. Además el paciente curso con pericarditis purulenta por MSSA, entidad infrecuente en la era antibiótica actual, y si bien es cierto, las lesiones por vecindad pulmonar y mediastinal son los puntos de partida más frecuentes, la bacteriemia también lo es; los estudios reportan que Staphylococcus aureus es el germen más frecuente $(11,12)$.
Con los hallazgos clínicos y de laboratorio se sospechó de endocarditis infecciosa, sin embargo, la ecocardiografía transesofagica no mostró lesiones valvulares ni vegetaciones.

El tratamiento de soporte hemodinámico, ventilatorio se hizo precozmente dentro de la primera hora de admisión en la emergencia, lo cual ha demostrado disminuir la morbilidad y mortalidad en choque séptico; de igual manera los cultivos e inicio precoz de tratamiento antibiótico son muy importantes (13-15).

El manejo quirúrgico, los drenajes y desbridamiento de tejidos infectados, son fundamentales en el manejo del choque séptico. Nuestro paciente fue sometido a pericardiectomia subtotal, drenaje pleural y posterior liberación y decorticación pulmonar. El tratamiento antibiótico se realizó con oxacilina + vancomicina, con de escalación posterior a oxacilina luego del resultados de los cultivos. Con las estrategias mencionadas el paciente evolucionó de manera favorable y fue dado de alta vivo para seguir con tratamiento y controles.

Presentamos este caso para destacar la gravedad de la bacteriemia por Staphylococcus aureus meticilino sensible, con compromiso multiorgánico y así poder tomar medidas terapéuticas agresivas para disminuir la morbilidad y mortalidad por el mismo.

\section{Declaración de financiamiento y de conflictos de interés:}

El reporte fue financiado por los autores, quienes declaran no tener conflictos de interés. 


\section{Contribución de autoría:}

JZ: Desarrollo de la idea, redacción del reporte; aprobación de la versión final a ser publicada. VS: Acopio de la información del caso, redacción del reporte. LG, ER, AH: Revisión crítica del artículo; SY: Imágenes y fotografías.

\section{Correspondencia:}

Jaime Zegarra Piérola

Correo electrónico: jaime.zegarra.p@upch.pe

\section{REFERENCIAS BIBLIOGRÁFICAS}

1. García J, Agüero J, Parra J, Santos M. Enfermedades infecciosas. Concepto. Clasificación. Aspectos generales y específicos de las infecciones. Criterios de sospecha de enfermedad infecciosa. Pruebas diagnósticas complementarias. Criterios de indicación. Medicine (Madr). 2010; 10(49):32513264. doi: 10.1016/S0304-5412(10)70027-5.

2. Lodise T, Graves J, Evans A, et al. Relationship between Vancomycin MIC and failure among patients with methicillin-resistant Staphylococcus aureus bacteremia treated with vancomycin. Antimocrob Agents and Chemother. 2008; 52(9):3315-3320.

3. Wang J, Chen S, Wang JT, et al. Comparison of both clinical features and mortality risk associated with bacteremia due to community acquired methicillin-resistant Staphylococcus aureus and methicillin-susceptible $S$. aureus. Clin Infect Dis. 2008; 46:799-806.

4. van-Hal SJ, Jensen SO, Vaska V, et al. Predictors of Mortality in Staphylococcus aureus Bacteremia. Clin Microbiol Rev. 2012; 25(2):362-86. doi: 10.1128/CMR.05022-11.
5. Corey GR. Staphylococcus aureus Bloodstream Infections: Definitions and Treatment. Clin Infect Dis. 2009; 48 Suppl 4:S254-9. doi: 10.1086/598186

6. Nabe C. Staphylococcus aureus bacteremia: epidemiology, pathophysiology, and management strategies. Clin Infect Dis. 2009; 48:231-7.

7. Martin G. The Epidemiology of Sepsis in the United States from 1979 through 2000. N Engl J Med. 2003; 348:1546-54.

8. Ranieri VM, Thompson B, Barie P, et al. Drotrecogin Alfa (Activated) in Adults with Septic Shock. N Engl J Med. 2012; 366:2055-64.

9. De-Backer D. Comparison of dopamine and norepinephrine in the treatment of shock. $\mathrm{N}$ Engl $\mathrm{J}$ Med. 2010; 362:779-89.

10. SelfW, Wunderink R, Williams D, etal.Staphylococcus aureus Community-acquired Pneumonia: Prevalence, Clinical Characteristics, and Outcomes. Clin Infect Dis. 2016; 63(3):300-309.

11. Klacsmann P. The changed spectrum of purulent pericarditis: An 86-year autopsy experience in 200 patients. Am J Med. 1977; 63(5):666-73. doi: 10.1016/0002-9343(77)90150-4

12. Gabler M. Cardiac tamponade secondary to purulente pericarditis diagnosed with the aid of emergency department ultrasound. Am J Emerg Med. 2017; 35(8):1212.e1-1212.e3

13. Levy M. The surviving sepsis campaign bundle: 2018 Update. Intensive Care Med. 2018; 44:925-928.

14. Dellinger RP, Levy MM, Rhodes A, et al. Surviving sepsis campaign: International guidelines for management of severe sepsis and septic shock: 2012. Crit Care Med. 2013; 41:580-637.

15. Levy MM, Rhodes A, Phillips GS, et al. Surviving Sepsis Campaign: Association between performance metrics and outcomes in a 7.5-year study. Crit Care Med. 2015; 43:3-12.

Recibido: 20/10/2019

Aceptado: 10/08/2020 